\title{
Article
}

\section{Examining the influence of grip type on wrist and club head kinematics during the golf swing: Benefits of a local co- ordinate system}

Carson, H.J., Richards, J., and Mazuquin, B.

Available at http://clok.uclan.ac.uk/23700/

Carson, H.J. ORCID: 0000-0002-3785-606X, Richards, J., ORCID: 0000-00024004-3115 and Mazuquin, B. (2019) Examining the influence of grip type on wrist and club head kinematics during the golf swing: Benefits of a local coordinate system. European Journal of Sport Science, 19 (3). pp. 327-335. ISSN 1746-1391

It is advisable to refer to the publisher's version if you intend to cite from the work. http://dx.doi.org/10.1080/17461391.2018.1508504

For more information about UCLan's research in this area go to http://www.uclan.ac.uk/researchgroups/ and search for <name of research Group>.

For information about Research generally at UCLan please go to http://www.uclan.ac.uk/research/

All outputs in CLoK are protected by Intellectual Property Rights law, including Copyright law. Copyright, IPR and Moral Rights for the works on this site are retained by the individual authors and/or other copyright owners. Terms and conditions for use of this material are defined in the policies page. 
Examining the Influence of Grip Type on Wrist and Club Head Kinematics during the Golf Swing: Benefits of a Local Co-ordinate System

Howie J. Carson ${ }^{*}$, Jim Richards ${ }^{2}$ and Bruno Mazuquin ${ }^{3}$

${ }^{1}$ Institute for Coaching and Performance, University of Central Lancashire, Preston, United Kingdom

${ }^{2}$ Allied Health Research Unit, University of Central Lancashire, Preston, United Kingdom

${ }^{3}$ Warwick Clinical Trials Unit, University of Warwick, United Kingdom

*Correspondence concerning this article should be addressed to Howie J. Carson, 006

Greenbank Building, Institute for Coaching and Performance, University of Central

Lancashire, Preston, PR1 2HE. E-mail: HCarson1@uclan.ac.uk 
Abstract

2 Wrists movements have been identified as an important factor in producing a successful golf

3 swing, with their complex motion influencing both club head velocity and orientation.

4 However, a detailed analysis of wrist angles is lacking in the literature. The purpose of this

5 study was to determine kinematics across wrists and club head characteristics during the golf

6 swing under weak, neutral and strong grip conditions. Twelve professional male golfers

7 executed 24 shots using a driver under three grip conditions. A six degrees of freedom

8 analysis of the hand with respect to the distal forearm was performed using a 10-camera

9 three-dimensional motion capture system. Differences in joint angles were explored using

10 repeated measures ANOVAs at key swing events (onset, top of backswing and impact), in

11 addition club head velocity and clubface angle at impact were also explored. Main findings

12 revealed significant differences in flexion/extension and internal/external rotation for both

13 wrists at all swing events, whereas fewer significant interactions were found in ulnar/radial

14 deviation across grips for both wrists at all events. Clubface angle only differed significantly

15 between the weak and the strong and neural grips, presenting a more 'open' clubface to the

16 intended hitting direction. This study is the first to explore tri-planar wrist movement and the

17 effect of different grips, such analysis has implications for coaching knowledge and practice

18 and should inform future research into different aspects of skill, technique analysis and may

19 inform injury mechanisms/prevention.

Keywords: driver, golf, Qualisys, internal/external rotation, range of motion, six degrees of freedom analysis. 
Examining the Influence of Different Grip Types on Wrist and Club Head Kinematics during the Golf Swing: Benefits of a Local Co-ordinate System

Wrists movements have been identified as an important factor in the production of a successful golf swing, with their complex range of motion (ROM) influencing both club head velocity and orientation (Nesbit, 2005; PGA, 2008; Sprigings \& Neal, 2000). They have also been identified as having the greatest angular velocities of all joints during the golf swing (Zheng, Barrentine, Fleisig, \& Andrews, 2008) and are consistently reported as the primary injury site, particularly in the lead wrist (left in right-handed golfers), amongst high-level golfers (Barclay, West, Shoaib, Morrissey, \& Langdown, 2011; McCarroll, Retting, \& Shelbourne, 1990). For example, Barclay et al. reported within an international survey of 526 club and touring professionals a $66 \%$ prevalence of injury and within that sample a $44 \%$ incidence rate pertaining to the wrist. Therefore, it is important that sport practitioners are able to understand the nature of high-level golfers' lead and trail wrist kinematics during the golf swing. Consequently, this may offer a useful insight into the mechanisms of wrist injuries and a more detailed understanding of technique effectiveness.

To date, studies reporting three-dimensional wrist kinematics have been either forward dynamic (MacKenzie \& Sprigings, 2009; Sprigings \& Neal, 2000) or experimental (Cahalan, Cooney III, Tamai, \& Chao, 1991; Fedorcik, Queen, Abbey, Moorman Iii, \& Ruch, 2012; Zheng et al., 2008). However, little data exists on high- or elite-level golfers. Two studies that have reported findings from high-level participants are Zheng et al. (2008) and Fedorcik et al. (2012). Despite inclusion of high-level participants, data reported does not allow a complete analysis of wrist mechanics. Zheng et al. (2008) defined the wrist by the golf club shaft moving relative to the forearm, which is unlikely to provide a complete understanding about the three-dimensional movement patterns. This would also partly explain why previous data only exists in one or two axes of rotation; ulnar/radial deviation 
and flexion/extension (Fedorcik et al., 2012; Nesbit, 2005; Zheng et al., 2008). Further investigating the wrists' three-dimensional movement patterns could prove beneficial in understanding different strategies and their relationship to golf swing effectiveness.

Indeed, non-sporting studies have previously reported ROM in internal/external rotation about the wrist joint independently of forearm pronation/supination at the radioulnar joint. Gilmour, Richards and Redfern (2012) examined wrist kinematics during activities of daily living (ADL; e.g., opening/closing jars). Results from 9 healthy participants, which were reported and published as part of a conference proceeding, revealed a maximum mean ROM of $31.7^{\circ}$. Indeed, this finding is consistent with other studies using simulated ADL, where a mean radiometacarpal internal/external rotation (ROM) of $34.1^{\circ}$ was reported (Gupta \& Moosawi, 2005). Notably, it is acknowledged that wrist joint internal/external rotation is passively controlled (i.e., voluntary forearm rotation does not independently axially rotate the wrist joint) when performing ADL and external resistance is applied. In Gilmour et al.'s (2012) study, resistance was applied by the objects being manipulated; and Gupta and Moosawi (2005) actively forced rotation of the forearm by fixing the position of the phalanges. It is likely that the inertial moments caused by the club accelerations during the golf swing and/or the hands' orientation when gripping the handle, may also result in such rotation. Therefore, wrist joint internal/external rotation should be included in future threedimensional analyses to allow for greater understanding.

Furthermore, existing research is limited by the amount of data provided during the golf swing. Previous studies have only reported data at specific events such as the top of the backswing and impact (e.g., Zheng et al., 2008). Despite this, studies have identified a common feature for the lead wrist amongst high-level golfers when compared to novices.

Data indicate high-level golfers to be more radially deviated at the top of the backswing, coupled with a delayed transition to ulnar deviation during the downswing until impact 
74 (Lindsay, Mantrop, \& Vandervoort, 2008; Sharp, 2009; Sprigings \& Neal, 2000). According

to the Training Academy of The Professional Golfers' Association (PGA) of Great Britain and Ireland, these events represent the swing principle 'release,' which describes returning the clubface back in line with the target through the "impact position while freeing the power created in the backswing" (PGA, 2008, p. 48), which are important to both distance and accuracy. However, what appears to be lacking in the literature is a detailed threedimensional analysis for both wrists during the entire golf swing and their relationship with club head measures at impact, and the effect of different grip types, often described by the address position. For example, a 'strong' grip presents the palm of the lead hand more on top of the handle and the trail hand more underneath, versus a 'weak' grip with the palm of the lead hand rotated anticlockwise around the handle and the trail hand more on top (see Figure 1). A weak grip (and vice versa for a strong grip) is described as such due to its apparent limiting influence on wrist 'action'/release, therefore reducing ball carry distance (Najar, 2010). Furthermore, golf coaching texts explain that the direction of clubface alignment at impact, relative to the intended target line, can be associated with grip type (PGA, 2010), which can be inferred by the extent of lead wrist flexion/extension at the top of the backswing. Addressing the latter, greater extension indicates a likely 'open' clubface and flexion a likely 'closed' clubface (Haney, 2012; PGA, 2008). Consequently, it is possible that some golfers may attempt adjustments to their grip to facilitate different shot shapes. If a complete three-dimensional analysis of the wrist joints were able to provide increased detail across the three planes of motion, it may be possible to assess for any exact changes in the wrist kinematics as a result of different starting grip techniques.

$$
* * * * \text { Figure } 1 \text { here } * * * *
$$


Therefore, the purpose of this study was twofold; firstly, to determine kinematics across both wrists during the golf swing when employing a three-dimensional analysis, and; secondly, to assess for any changes in club head characteristics at impact resulting from short-term (within session) grip modification under weak, neutral and strong grip conditions.

\section{Method}

\section{Participants}

Twelve right-handed male golfers $\left(M_{\mathrm{age}}=32 \pm 9.3\right.$ years $)$ were recruited for this study. All were PGA Professional golf coaches which meant that they did not have a

107 handicap but would have required a maximum handicap of 4 prior to attaining professional

108 status. Therefore, all golfers can be considered as highly skilled. Preceding data collection,

109 participants were required to read an information sheet and provide informed consent.

110 Ethical approval was gained from the University’s Ethics Committee prior to data collection.

111 Participant eligibility required no current or prior wrist injuries as assessed through self-

112 report.

\section{Procedures}

114 Participants warmed-up using self-conducted exercises and practice tee shots from an 115 artificial turf mat using their own driver and wearing golf shoes. Three blocks of eight full 116 swing executions were completed, requiring a squash ball to be hit with participants' own

117 driver towards a vertical target fixed on the laboratory wall approximately $15 \mathrm{~m}$ away. The

118 first block required a natural and individually-preferred grip, therefore allowing the capture

119 of participants most well-established movement patterns (Carson \& Collins, 2016). Three

120 participants had a naturally strong grip, seven a neutral grip and two a weak grip. Two repeated blocks then followed to satisfy the remaining grip conditions in a randomly assigned

122 order. Grip manipulations were visually checked to ensure adequate understanding; all

123 participants adhered to the task requirements at this stage by displaying the correct number of 
124 knuckles on each hand at the address position as shown in Figure 1. Accordingly, eight full swing executions were captured from each participant utilising a neutral, strong and weak

126 grip technique.

127 Kinematic data were collected using 10 Oqus 700 cameras (Qualisys Medical AB, 128 Sweden) at a sampling rate of $300 \mathrm{~Hz}$. Qualisys Track Manager ${ }^{\mathrm{TM}}$ (QTM, Version 2.11,

129 Qualisys Medical Ltd., Sweden) was used to reconstruct the three-dimensional co-ordinates 130 of $10 \mathrm{~mm}$ passive retro-reflective markers applied bilaterally to the following anatomical 131 sites: medial and lateral humerus epicondyles, radial and ulnar styloid processes and $2^{\text {nd }}$ and

$1325^{\text {th }}$ metacarpal heads. Rigid clusters were positioned on the distal forearms and dorsum of the 133 hands allowed segmental tracking in six degrees-of-freedom. Seven $6 \mathrm{~mm}$ markers were 134 positioned on the four extremities of the clubface and three on the club head; the ball was 135 also marked with retro-reflective tape. Four $10 \mathrm{~mm}$ passive retro-reflective markers were 136 affixed onto the artificial turf mat in a cross formation to enable club head orientation and 137 velocity to be calculated (Figure 2). A neutral static calibration trial was captured prior to 138 testing with the participant adopting the anatomical position for $1 \mathrm{~s}$, markers positioned at 139 anatomical landmarks were subsequently removed prior to golf swing executions.

\section{Data Processing}

Raw kinematic data for a minimum of five trials from each condition per participant were exported into c3d file format and analysed using Visual 3D v5.01.25 software (CMotion Inc., USA). Co-ordinate systems were assigned using joint centres defined by the

147 medial and lateral markers on the proximal and distal aspects for each segment using a single 148 frame of the static calibration trial $(y$-axis $=$ anterior-posterior, $x$-axis $=$ medial-lateral and $z$ - 
149 axis = proximal-distal). The radioulnar segments were defined proximally using the medial

150 and lateral humerus epicondyles and distally using the radial and ulnar styloid processes. The

151 hands were defined proximally using the radial and ulnar styloid processes and distally using

152 the $2^{\text {nd }}$ and $5^{\text {th }}$ metacarpal heads. Wrist joint angles were calculated in all three axes of

153 rotation of the distal segment relative to the local co-ordinate system (LCS) of the proximal

154 segment, using an $\mathrm{X}$ (flexion/extension), Y (medial/lateral), Z (axial) Cardan sequence as

155 previously employed within golf research to measure wrist mechanics (Joyce, Burnett,

156 Cochrane, \& Reyes, 2016; Sinclair, Currigan, Fewtrell, \& Taylor, 2014), and is an equivalent

157 Cardan sequence recommended by (Wu et al., 2005). Movement in extension, radial

158 deviation and external rotation were defined as positive and flexion, ulnar deviation and

159 internal rotation were defined as negative. The club head was defined proximally using the

160 two superior markers on the clubface, with the marker closest to the shaft as the medial and

161 the other as lateral; inferior clubface markers were used to define the clubface distally, again

162 with the marker closest to the shaft as the medial and that furthest away as lateral. To

163 ascertain the clubface angle at impact, the club head angle was referenced in the $z$-axis of the

164 cross segment on the mat (positive values depicting a clubface pointing left of the ball-to-

165 target line and negative values to the right of the ball-to-target line), in addition club head

166 velocity was calculated at impact. Data were filtered using a low-pass Butterworth filter with

167 a cut off frequency of $25 \mathrm{~Hz}$.

168 Four events were identified and used to divide the swing into three phases, with the

169 time between each event normalised to 101 points. "Onset" was defined when the club head

170 linear speed crossed a threshold value of $0.0 \mathrm{~m} / \mathrm{s}$ in the global $x$-axis on swing ascent. "Top"

171 was defined when the club head linear speed reached its lowest negative value in the global $z_{-}^{-}$

172 axis prior to swing decent. "Impact" was defined immediately before the ball recorded a 
173 positive velocity. Finally, "Follow through" was defined when the left hand linear speed

174 crossed a threshold of $0.0 \mathrm{~m} / \mathrm{s}$ in the global $x$-axis following the impact event.

\section{Statistical Analysis}

Data were analysed using SPSS Statistics 23.0 (IBM Corporation, USA) software.

177 Repeated measures ANOVAs were used to test for differences between wrist joint angles at

178 the swing onset, top and impact events, maximum and minimum angles, ROM and clubface

179 angle and velocity at impact. Main effects were assessed using the Greenhouse-Geisser

180 correction when Mauchly's sphericity test was violated and effect sizes were provided

181 through the partial eta-squared $\left(\eta_{\mathrm{p}}^{2}\right)$ statistic. Post hoc pairwise comparisons were made

182 using the Bonferroni test when appropriate. A $P$-value $<0.05$ was considered as significant

183 for all statistical tests.

Golf swing wrist kinematics (means and standard deviations) for all grip types are shown in Table 1. The following details any significant findings.

187 Joint Angles at Identified Events

188 Onset. While it could not be predetermined based on previous empirical study exactly how the wrist joint would differ, it was important to test for at least some level of change to support the visual manipulation checks employed. For the left wrist, there were main effects with large effect sizes for grip type on flexion/extension, $P<0.001, \eta_{\mathrm{p}}{ }^{2}=0.78$,

192 and internal/external rotation, $P<0.001, \eta_{\mathrm{p}}^{2}=0.73$, angles, with significant differences

193 evident in flexion/extension between neutral and weak $(P=0.001)$, neutral and strong $(P=$ 194 0.002) and strong and weak $(P<0.001)$ grips and for internal/external rotation between neutral and weak $(P<0.001)$, neutral and strong $(P=0.029)$ and strong and weak $(P<0.001)$ grips. Similarly for the right wrist, main effects with large effect sizes for grip type were

197 revealed in flexion/extension, $P<0.001, \eta_{\mathrm{p}}^{2}=0.78$ and internal rotation, $P<0.001, \eta_{\mathrm{p}}{ }^{2}=$ 
1980.73 , but also ulnar/radial deviation with a medium effect size, $P=0.018, \eta_{\mathrm{p}}{ }^{2}=0.37$. Post

199 hoc analyses revealed significant differences in flexion/extension between neutral and weak

$200 \quad(P=0.003)$, neutral and strong $(P<0.001)$, and strong and weak $(P<0.001)$ grips, in

201 internal/external rotation between neutral and weak $(P=0.001)$ and strong and weak $(P<$

$2020.001)$ grips, with neutral and strong closely approaching significance $(P=0.055)$. No

203 significant differences were found in right wrist ulnar/radial deviation although the

204 differences between neutral and weak $(P=0.088)$ and weak and strong $(P=0.061)$ showed a

205 trend towards significance.

Top. Data at the top of the swing reveal that onset differences were not always

207 consistent. For the left wrist, there were significant main effects with a large effect size for

208 grip type on flexion/extension, $P<0.001, \eta_{\mathrm{p}}{ }^{2}=0.70$, and a medium effect size for

209 internal/external rotation, $P=0.008, \eta_{\mathrm{p}}^{2}=0.35$, angles. Significant differences were shown

210 in flexion/extension between neutral and strong $(P<0.001)$ and weak and strong $(P=0.001)$

211 grips, with neutral and weak grips only approaching significance $(P=0.07)$, and for

212 internal/external rotation between strong and weak grips $(P=0.036)$. Right wrist kinematics

213 showed main effects with a large effect size for grip type on flexion/extension, $P=0.002, \eta_{\mathrm{p}}{ }^{2}$

$214=0.43$, and medium effect sizes for ulnar/radial deviation, $P=0.022, \eta_{\mathrm{p}}{ }^{2}=0.37$, and internal

215 rotation, $P=0.03, \eta_{\mathrm{p}}{ }^{2}=0.27$. Post hoc tests revealed significant differences in

216 flexion/extension between weak and strong $(P=0.005)$ grips and in ulnar/radial deviation

217 between neutral and strong $(P=0.02)$ and weak and strong $(P=0.045)$ grips. No significant

218 differences between grips were shown for internal/rotation angles.

219 Impact. There were significant main effects with large effect sizes for left wrist grip

220 type on flexion/extension, $P=0.002, \eta_{\mathrm{p}}^{2}=0.57$, and internal/external rotation, $P=0.003, \eta_{\mathrm{p}}{ }^{2}$

$221=0.49$, angles. Significant differences were shown in flexion/extension between neutral and

222 strong $(P=0.019)$, neutral and weak $(P=0.011)$ and weak and strong $(P=0.006)$ grips, and 
223 for internal/external rotation between neutral and strong $(P=0.029)$ and strong and weak $(P$

$224=0.014)$ grips. Right wrist kinematics showed main effects with large effect sizes for grip

225 type on flexion/extension, $F(2,22)=8.98, P=0.001, \eta_{\mathrm{p}}{ }^{2}=0.45$, ulnar/radial deviation, $P=$

$2260.002, \eta_{\mathrm{p}}{ }^{2}=0.43$, and a medium effect size for internal rotation, $P=0.004, \eta_{\mathrm{p}}{ }^{2}=0.39$. Post

227 hoc analyses revealed significant differences between weak and strong grips in

228 flexion/extension $(P=0.001)$, ulnar/radial deviation $(P=0.02)$ and internal/external rotation

$229(P=0.012)$ grips.

230 Minimum/Maximum Angles and Range of Motion

When analysing the entire golf swings from the onset to follow-through events, there

was a significant main effect with large effect size of grip type on left wrist minimum

233 flexion/extension, $P=0.001, \eta_{\mathrm{p}}{ }^{2}=0.64$, and medium effect size for internal/external rotation, $P=0.009, \eta_{\mathrm{p}}{ }^{2}=0.35$, angles. Post hoc tests showed significant differences within

flexion/extension between neutral and strong $(P<0.001)$ and strong and weak $(P=0.002)$

grips and within internal/external rotation there was a trend towards significance between neutral and strong $(P=0.068)$ and strong and weak $(P=0.057)$ grips. There was a significant main effect with a medium effect size of grip type on right wrist minimum internal/external rotation angle, $P=0.027, \eta_{\mathrm{p}}^{2}=0.34$, but post hoc tests showed no significant differences between each of the grips. Right wrist minimum ulnar/radial deviation only tended towards significance, $P=0.051$. There was a significant main effect with medium effect size of grip type on left wrist maximum flexion/extension, $P=0.002, \eta_{\mathrm{p}}^{2}=$

243 0.34. Post hoc tests showed significant differences between neutral and strong $(P=0.012)$.

244 There was a significant main effect with large effect size of grip type on right wrist maximum

245 flexion/extension angle, $P=0.007, \eta_{\mathrm{p}}{ }^{2}=0.43$. Right wrist maximum internal/external

246 rotation approached significance, $P=0.064$. Post hoc tests revealed significant differences

247 for flexion/extension between strong and weak $(P=0.016)$, with neutral and strong grips 
248 almost reaching significance $(P=0.05)$. Despite these differences in minimum and maximum angles, overall ROM appeared to be relatively unaffected. There was only a significant main effect with medium effect size of grip type on left wrist flexion/extension ROM, $P=.045, \eta_{\mathrm{p}}^{2}=0.29$. However, post hoc analyses revealed nonsignificant results.

\section{Club Kinematics at Impact}

There was a significant main effect with a large effect size of grip type on clubface angle, $P=0.001, \eta_{\mathrm{p}}^{2}=0.47$. As expected, the neutral grip clubface angle was between the angles for strong and weak grips. Notably, all clubfaces were presented to the same side relative to the ball-target line, to the right (Table 2). Significant differences, however, were only found between neutral and weak $(P=0.019)$ and strong and weak $(P=0.011)$ grips. There was no significant main effect found for grip type on club head velocity, $P=0.301$.

\section{Discussion}

This study addressed methodological shortcomings of previous research into golf wrist mechanics by employing a three-dimensional analysis using anatomical LCSs.

268 Furthermore, it compared several club head kinematics at impact resulting from purposeful, 269 albeit acute, modifications to grip type within a sample of high-level golfers. Wrist movement was tri-planar in nature, indicating greater complexity than previously reported

271 (Cahalan et al., 1991; Fedorcik et al., 2012; Zheng et al., 2008). While this method is not 272 always appropriate for golf swing analyses (e.g., when analysing general timing), it is 
important to recognise that simplistic wrist analyses could ignore important movement patterns.

Regarding internal/external rotation, mean trail wrist ROM was similar to previous data (Gilmour et al., 2012; Gupta \& Moosawi, 2005). Lead wrist mean ROM, however, was much higher. Internal rotation was similar between wrists, indicating that additional external rotation accounted for this difference. Considering the lead wrist's injury prevalence in highlevel golfers, this subtle difference could be a contributing factor. Moreover, from the address position the lead wrist was closest to its maximum internal rotation angle, which is also likely to persist for the longest duration as the golfer sets up and prepares to execute the shot. Although currently speculative in nature, the tri-planar data certainly appears able to provide additional detail to begin exploring specific questions about golf swing technique and the underlying causes of performance. Similarly, researchers exploring the 'X-factor' principle have recently advocated the necessity for an anatomical LCS to gain a greater biomechanical meaning (Brown, Selbie, \& Wallace, 2013). Other factors that might interact with this wrist movement to result in injury include the nature of club-ground contact and intensity of practice undertaken. At present, however, we await further investigations along these lines.

Looking beyond the novel internal/external rotation data, the nonsignificant differences in club head velocity suggests that any differential in observed shot distance between grip types may not be due to the transfer of energy to the club head. Instead, underpinning causes could reside with precision elements; for instance, clubface loft, angle in relation to the swing direction and, therefore, resultant ball trajectory. Further support for this can be inferred from the trail wrist flexion/extension ROM during the downswingwhich is indicative of angular velocity and directly related to the amount of power applied (Sinclair et al., 2014)—showing very little/no change across the three grip conditions. 
298 Differences between the tri-planar angles were however evident at impact. As such, it is

299 possible that the type rather than the amount of movement needs further consideration when

300 examining the golf swing 'release' principle (Najar, 2010). From these data and for this

301 sample at least, simply changing the grip position does not appear beneficial to increasing

302 club head velocity.

303 Top of the backswing data are also of interest. Specifically, the mean lead wrist was

304 in extension irrespective of grip type and all club face angles were aligned to the right of the

305 shot direction line (open) at impact. Notably, this is somewhat contrary to Haney's (2012)

306 explanation that the wrist angle at the top of the backswing, and subsequent impact

307 orientation, could relate to grip. As a possible interpretation, these high-level golfers were

308 able to resist the 'likelihood' of closing the clubface at impact with a strong grip, maintaining

309 a relatively square position, whereas this was comparatively more challenging with a weak

310 grip. This supports PGA's (2010) suggestion that golfers tend closer towards a strong rather

311 than weak grip. Indeed, most participants expressed a preference for either a neutral or

312 strong grip during debriefs that followed the trials. It is perhaps, therefore, unsurprising that

313 the strong grip could be more functionally adapted compared to the weak grip, due to

314 increased familiarity and comfort in the executions.

315 Moreover, regarding individual differences, despite Table 1 showing strong-neutral-

316 weak grips resulted in a fairly consistent and ordered ascending/descending sequence of

317 angles for the variables, some showed no difference across conditions. Notably, upon

318 inspection of individual data, no single participant entirely matched these ordered sequences

319 from the group data. As such, this supports the rationale for individual technical analyses

320 within coaching practice (Brown et al., 2011; Kostrubiec, Zanone, Fuchs, \& Kelso, 2012).

321 Undoubtedly, some movements will be similar across participants, therefore abiding by a

322 general technical template. However, coaches should be cautious when constructing 
individualised mental models of performance not to fall into the 'flaw of average' heuristic trap (Rose, 2016) when assessing many swing variables. In short, the idea that a mental model of performance should target the average of skilled/elite players (e.g., Mann \& Griffin, 1998), even if 'windows' around the mean are catered for (Rose, 2016), is inevitably suboptimal at best.

In addition to an improved understanding of mechanics by employing LCSs, there are also pragmatic advantages to be realised. Specifically, this arises when requiring longitudinal analyses, such as when diagnosing and monitoring technique during skill refinement (Carson \& Collins, 2011). Using more commonly employed global co-ordinate systems in the lab and applied settings (e.g., a fixed camera positioned in the sagittal or coronal plane) cannot guarantee the exact relative positioning between the golfer and co-ordinate system axes between sessions. Consequently, intersession comparisons are less reliable and have greater planar cross-talk, with LCSs suffering fewer inconsistencies in measurement; data are less affected by variations across trials, days and environments.

Despite methods employed in this study, limitations must be recognised. Technique variations have been reported across different golf clubs (Egret, Vincent, Weber, Dujardin, \& Chollet, 2003), especially when executing from the ground and not a tee. Further understanding would therefore derive from employing LCSs beyond the sole use of a driver. From a motor control perspective, issues of ecological validity are also noteworthy in that the laboratory environment is unrepresentative of golf course conditions (Pinder, Davids, Renshaw, \& Araújo, 2011). It has been reported that changes in automaticity can occur following the removal of naturalistic features (Carson, Collins, \& Richards, 2016), however we cannot say in this case whether kinematics were compromised in any way. Mobile technologies that permit motion capture on the golf course may be able to overcome this limitation in future investigations. Relatedly, when considering participants' high skill status, 
two of the grip conditions were less familiar/comfortable and therefore reflect a short-term perturbation to technique which we would expect to be disruptive of control processes

350 (Charlton \& Starkey, 2011). Accordingly, we recommend caution in assuming that any differences truly represent well-established techniques. Future research may extend this novel methodology by testing between individuals with different preferable grip types and collecting valuable ball flight data to enhance our understanding of the relationship with performance outcomes. Finally, addressing the collection and processing of kinematic data, this study defined the hand as a rigid segment and was able to detect differences within that segment relative to the forearm, however a more detailed analysis of the structures within the hand maybe possible (Gupta \& Moosawi, 2005), which may yield a greater understanding of the movement and injury risks during the golf swing. Additionally, while Joyce, Burnett, and Ball (2010) determined that different joint angles for the trunk resulted from different Cardan sequences, it is important to highlight that no research has yet investigated any such differences when assessing wrist motion.

\section{Conclusion}

This paper extends current knowledge relating to the lead and trail wrist mechanics during the golf swing, through use of anatomical LCSs. Specifically, its contribution can be seen in the identification of movement in internal/external rotation and the interpretation of data from a coaching perspective. It is hoped that the methods employed in this study can be used to inform future research into many aspects of skill, technique analysis and skill

368 development, and provided a greater understanding of injury mechanisms and their prevention.

\section{Disclosure Statement}

372 There is no potential conflict of interest concerning this non-funded research. 


\section{References}

Barclay, C., West, S., Shoaib, Q., Morrissey, D., \& Langdown, B. (2011). Injuries patterns among professional golfers: An international survey. British Journal of Sports Medicine, 45, e1. doi:10.1136/bjsm.2010.081554.12

Brown, S. J., Nevill, A. M., Monk, S. A., Otto, S. R., Selbie, W. S., \& Wallace, E. S. (2011). Determination of the swing technique characteristics and performance outcome relationship in golf driving for low handicap female golfers. Journal of Sports Sciences, 29, 1483-1491. doi:10.1080/02640414.2011.605161

Brown, S. J., Selbie, W. S., \& Wallace, E. S. (2013). The X-Factor: An evaluation of common methods used to analyse major inter-segment kinematics during the golf swing. Journal of Sports Sciences, 31, 1156-1163. doi:10.1080/02640414.2013.775474

Cahalan, T. D., Cooney III, W. P., Tamai, K., \& Chao, E. Y. S. (1991). Biomechanics of the golf swing in players with pathologic conditions of the forearm, wrist, and hand. The American Journal of Sports Medicine, 19, 288-293. doi:10.1177/036354659101900314

Carson, H. J., \& Collins, D. (2011). Refining and regaining skills in fixation/diversification stage performers: The Five-A Model. International Review of Sport and Exercise Psychology, 4, 146-167. doi:10.1080/1750984x.2011.613682

Carson, H. J., \& Collins, D. (2016). The fourth dimension: A motoric perspective on the anxiety-performance relationship. International Review of Sport and Exercise Psychology, 9, 1-21. doi:10.1080/1750984X.2015.1072231

Carson, H. J., Collins, D., \& Richards, J. (2016). Initiating technical refinements in high-level golfers: Evidence for contradictory procedures. European Journal of Sport Science, 16, 473-482. doi:10.1080/17461391.2015.1092586 
Charlton, S. G., \& Starkey, N. J. (2011). Driving without awareness: The effects of practice and automaticity on attention and driving. Transportation Research part F: Traffic Psychology and Behaviour, 14, 456-471. doi:10.1016/j.trf.2011.04.010

Egret, C. I., Vincent, O., Weber, J., Dujardin, F. H., \& Chollet, D. (2003). Analysis of 3D kinematics concerning three different clubs in golf swing. International Journal of Sports Medicine, 24, 465-470. doi:10.1055/s-2003-41175

Fedorcik, G. G., Queen, R. M., Abbey, A. N., Moorman Iii, C. T., \& Ruch, D. S. (2012). Differences in wrist mechanics during the golf swing based on golf handicap. Journal of Science and Medicine in Sport, 15, 250-254. doi:10.1016/j.jsams.2011.10.006

Gilmour, A., Richards, J., \& Redfern, D. R. M. (2012). Investigation of 3D planar wrist movements during activities of daily living. Journal of Bone \& Joint Surgery, British Volume, 94-B, 65

Gupta, A., \& Moosawi, N. A. (2005). How much can carpus rotate axially? An in vivo study. Clinical Biomechanics, 20, 172-176. doi:10.1016/j.clinbiomech.2004.09.014

Haney, H. (2012, 4 June). Check your left wrist. Golf Digest. Retrieved from http://www.golfdigest.com/story/hank-haney-left-wrist

Joyce, C., Burnett, A., \& Ball, K. (2010). Methodological considerations for the 3D measurement of the X-factor and lower trunk movement in golf. Sports Biomechanics, 9, 206-221

Joyce, C., Burnett, A., Cochrane, J., \& Reyes, A. (2016). A preliminary investigation of trunk and wrist kinematics when using drivers with different shaft properties. Sports Biomechanics, 15, 61-75. doi:10.1080/14763141.2015.1123764

Kostrubiec, V., Zanone, P.-G., Fuchs, A., \& Kelso, J. A. S. (2012). Beyond the blank slate: Routes to learning new coordination patterns depend on the intrinsic dynamics of the 

learner-experimental evidence and theoretical model. Frontiers in Human Neuroscience, 6, 1-14. doi:10.3389/fnhum.2012.00222

Lindsay, D., Mantrop, S., \& Vandervoort, A. (2008). A review of biomechanical differences between golfers of varied skill levels. International Journal of Sports Science and Coaching, 3(Supplement 1), 187-197. doi:10.1260/174795408785024117

MacKenzie, S. J., \& Sprigings, E. J. (2009). A three-dimensional forward dynamics model of the golf swing. Sports Engineering, 11, 165-175. doi:10.1007/s12283-009-0020-9

Mann, R., \& Griffin, F. (1998). Swing like a pro: The breakthrough scientific method of perfecting your golf swing. New York: Broadway Books.

McCarroll, J. R., Retting, A. C., \& Shelbourne, K. D. (1990). Injuries in the amateur golfer. The Physician and Sports Medicine, 18, 122-126

Najar, B. (2010, 11 November). Get a grip - The right grip for you. Retrieved from http://www.pga.com/golf-instruction/instruction-feature/fundamentals/get-grip-rightgrip

Nesbit, S. M. (2005). A three dimensional kinematic and kinetic study of the golf swing. Journal of Sports Science and Medicine, 4, 499-519

PGA. (2008). Study guide: Golf coaching I. United Kingdom: The Professional Golfers' Association.

PGA. (2010). Study guide: Introduction to golf coaching. Sutton Coldfield: The Professional Golfers' Association of Great Britain and Ireland.

Pinder, R. A., Davids, K., Renshaw, I., \& Araújo, D. (2011). Representative learning design and functionality of research and practice in sport. Journal of Sport and Exercise Psychology, 33, 146-155. doi:10.1123/jsep.33.1.146

Rose, T. (2016). The end of average: How we succeed in a world that values sameness. Canada: HarperCollins. 
Sharp, R. S. (2009). On the mechanics of the golf swing. Proceedings of The Royal Society A, 465, 551-570. doi:10.1098/rspa.2008.0304

Sinclair, J., Currigan, G., Fewtrell, D. J., \& Taylor, P. J. (2014). Biomechanical correlates of club-head velocity during the golf swing. International Journal of Performance Analysis in Sport, 14, 54-63

Sprigings, E. J., \& Neal, R. J. (2000). An insight into the importance of wrist torque in driving the golfball: A simulation study. Journal of Applied Biomechanics, 16, 356366. doi:10.1123/jab.16.4.356

Wu, G., van der Helm, F. C. T., Veeger, H. E. J., Makhsous, M., Van Roy, P., Anglin, C., .. . Buchholz, B. (2005). ISB recommendation on definitions of joint coordinate systems of various joints for the reporting of human joint motion-Part II: Shoulder, elbow, wrist and hand. Journal of Biomechanics, 38, 981-992. doi:10.1016/j.jbiomech.2004.05.042

Zheng, N., Barrentine, S. W., Fleisig, G. S., \& Andrews, J. R. (2008). Kinematic analysis of swing in pro and amateur golfers. International Journal of Sports Medicine, 29, 487493. doi:10.1055/s-2007-989229 
92 Figures

93

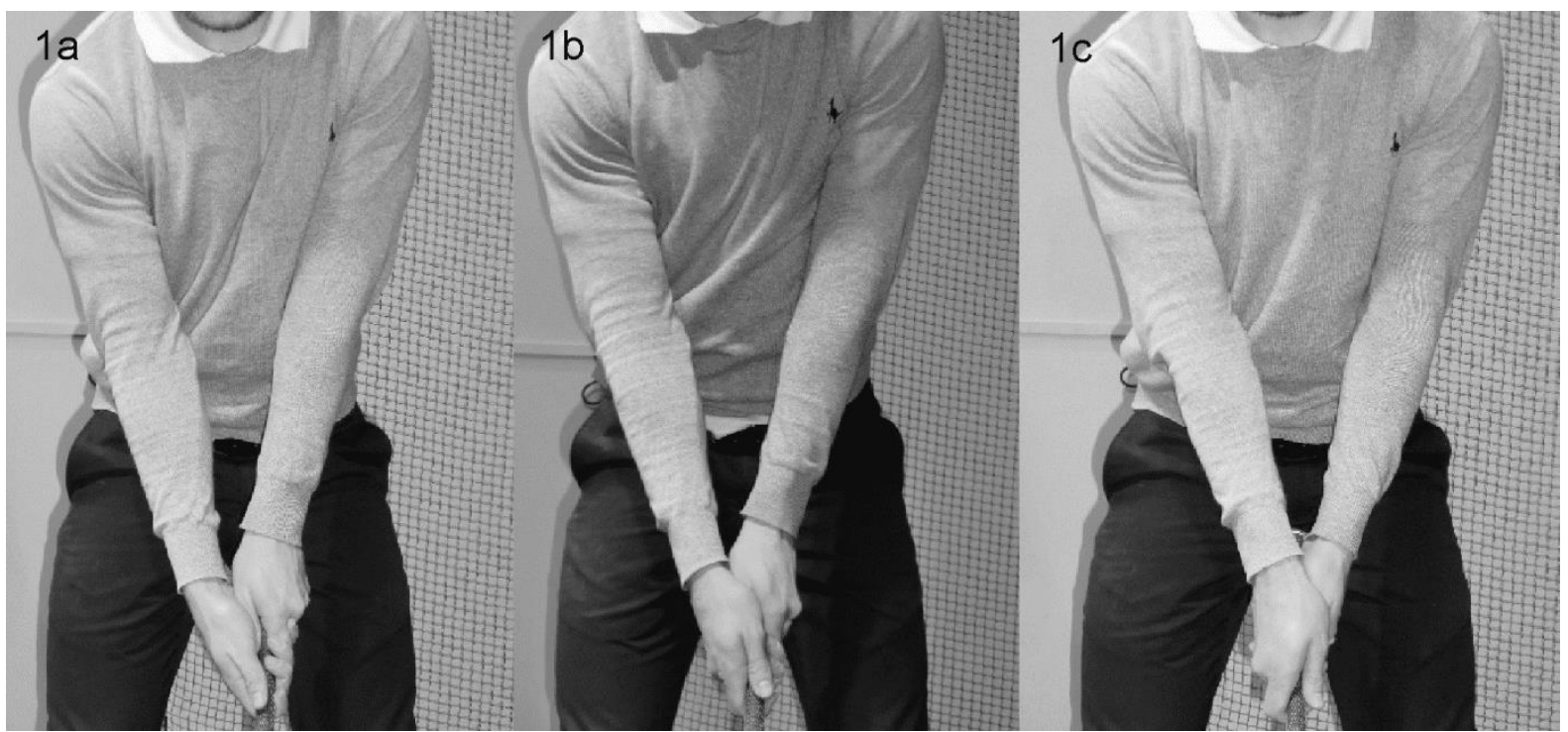

94 Figure 1. Different address grip positions viewed in the global coronal plane for a right-

95 handed golfer. The strong grip (1a) is characterised by the lead hand being positioned on top

96 of the handle with three knuckles shown to the golfer (first person perspective) and the trail

97 hand wrapped underneath with one knuckle shown. The neutral grip (1b) presents the golfer

98 with a view of two knuckles on each hand and the weak grip (1c) showing three knuckles on

99 the trail hand and one on the lead.

100

101

102 


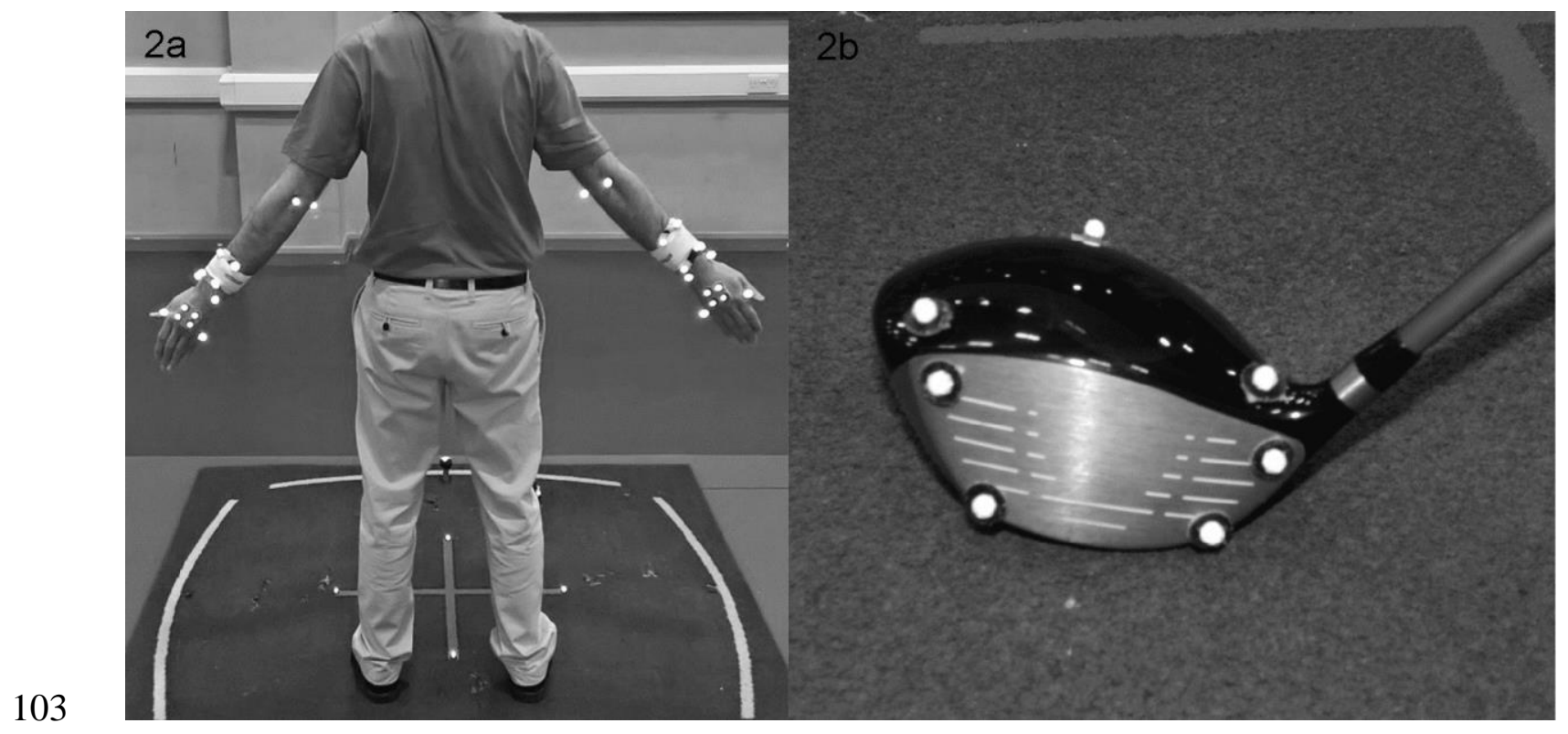

104 Figure 2. Anatomical and cluster marker placements on the forearm and hand segments, ball

105 marker and floor markers (2a). Club head and clubface marker placements (2b). 
Table 1. Lead and Trail Wrist Kinematics

\begin{tabular}{|c|c|c|c|c|c|c|}
\hline & \multicolumn{3}{|c|}{ Left (Lead) Wrist } & \multicolumn{3}{|c|}{ Right (Trail) Wrist } \\
\hline & Strong & Neutral & Weak & Strong & Neutral & Weak \\
\hline \multicolumn{7}{|l|}{ Swing Onset $\left({ }^{\circ}\right)$} \\
\hline Flexion/Extension & $33.8 \pm 10.0^{*}, * *$ & $29 \pm 10.9^{*, * * *}$ & $22.2 \pm 10.1^{* * * * * *}$ & $-2.2 \pm 4.7^{* *, * * *}$ & $3.8 \pm 5.4^{* * *}$ & $11.4 \pm 6.0^{*}, * * *$ \\
\hline $\begin{array}{l}\text { Ulnar/Radial } \\
\text { Deviation }\end{array}$ & $-15.2 \pm 11.7$ & $-15.0 \pm 11.1$ & $-15.8 \pm 10.7$ & $-22.6 \pm 8.8$ & $-23.6 \pm 8.9$ & $-24.7 \pm 8.8$ \\
\hline $\begin{array}{l}\text { Internal/External } \\
\text { Rotation }\end{array}$ & $-33.7 \pm 6.1^{* * * * * *}$ & $-29.9 \pm 6.3^{* * *}$ & $-24.6 \pm 5.3^{*, * * *}$ & $-13.0 \pm 7.4^{* *}$ & $-16.9 \pm 7.2^{*}$ & $-23.1 \pm 8.1^{*, * *}$ \\
\hline \multicolumn{7}{|l|}{ Top $\left(^{\circ}\right)$} \\
\hline Flexion/Extension & $14.4 \pm 12.1^{*, * *}$ & $6.6 \pm 11.8^{*}$ & $1.7 \pm 10.6^{* *}$ & $51.9 \pm 10.2^{*}$ & $54.0 \pm 11.3$ & $57.6 \pm 9.1^{*}$ \\
\hline $\begin{array}{l}\text { Ulnar/Radial } \\
\text { Deviation }\end{array}$ & $26.3 \pm 14.3$ & $24.3 \pm 12.6$ & $22.4 \pm 11.9$ & $26.4 \pm 6.2^{*, * *}$ & $24.8 \pm 6.5^{*}$ & $21.5 \pm 9.2^{* *}$ \\
\hline $\begin{array}{l}\text { Internal/External } \\
\text { Rotation }\end{array}$ & $-22.2 \pm 10.3^{*}$ & $-23.6 \pm 9.2$ & $-24.7 \pm 10.4^{*}$ & $-8.6 \pm 10.0$ & $-10.1 \pm 10.0$ & $-12.2 \pm 10.0$ \\
\hline \multicolumn{7}{|l|}{ Impact $\left(^{\circ}\right)$} \\
\hline Flexion/Extension & $8.9 \pm 10.5^{*, * *}$ & $5.5 \pm 12.5^{* * *}$ & $3.0 \pm 13.0^{* *, * * *}$ & $19.9 \pm 6.5^{*}$ & $21.3 \pm 6.9$ & $23.2 \pm 6.4^{*}$ \\
\hline $\begin{array}{l}\text { Ulnar/Radial } \\
\text { Deviation }\end{array}$ & $-24.2 \pm 7.7$ & $-24.6 \pm 8.7$ & $-25.3 \pm 9.3$ & $-22.7 \pm 9.3^{*}$ & $-24.1 \pm 9.9$ & $-25.7 \pm 11.0^{*}$ \\
\hline $\begin{array}{l}\text { Internal/External } \\
\text { Rotation }\end{array}$ & $-24.2 \pm 7.0^{*}, * *$ & $-21.4 \pm 7.1^{*}$ & $-19.0 \pm 8.5^{* *}$ & $-16.2 \pm 8.9^{*}$ & $-18.0 \pm 9.1$ & $-21.1 \pm 6.8^{*}$ \\
\hline \multicolumn{7}{|l|}{$\operatorname{ROM}\left(^{\circ}\right)$} \\
\hline Flexion/Extension & $59.76 \pm 14.3$ & $61.65 \pm 13.2$ & $64.11 \pm 12.9$ & $76.85 \pm 11.2$ & $77.85 \pm 10.2$ & $78.56 \pm 9.9$ \\
\hline $\begin{array}{l}\text { Ulnar/Radial } \\
\text { Deviation }\end{array}$ & $63.82 \pm 9.9$ & $63.36 \pm 10.0$ & $63.27 \pm 11.5$ & $72.73 \pm 7.5$ & $73.27 \pm 8.0$ & $72.97 \pm 7.7$ \\
\hline $\begin{array}{l}\text { Internal/External } \\
\text { Rotation }\end{array}$ & $45.77 \pm 9.6$ & $45.20 \pm 7.4$ & $44.5 \pm 8.7$ & $32.95 \pm 11.7$ & $32.01 \pm 11.3$ & $33.53 \pm 12.2$ \\
\hline
\end{tabular}


Minimum Angle $\left({ }^{\circ}\right)$

Flexion/Extension

Ulnar/Radial

Deviation

$-3.58 \pm 9.8^{*, * *}$

$-8.24 \pm 9.3^{*}$

$-11.56 \pm 7.4^{* *}$

$-18.19 \pm 9.3$

$-17.43 \pm 9.4$

$-15.33 \pm 9.9$

Internal/External

Rotation

$-29.03 \pm 8.2$

$-28.70 \pm 8.0$

$-28.80 \pm 8.1$

$-38.0 \pm 7.7$

$-38.70 \pm 7.3$

$-39.01 \pm 7.5$

$-39.02 \pm 8.8$

$-37.21 \pm 8.6$

$-36.47 \pm 8.7$

$-30.57 \pm 6.1$

$-30.58 \pm 6.0$

$-33.78 \pm 5.5$

Maximum Angle $\left(^{\circ}\right)$

Flexion/Extension

Ulnar/Radial

Deviation

$56.18 \pm 10.2^{*}$

$53.40 \pm 10.4^{*}$

$52.60 \pm 11.2$

$58.66 \pm 11.2^{*}$

$60.42 \pm 10.7$

$63.22 \pm 9.6^{*}$

$34.80 \pm 12.8$

$34.66 \pm 12.1$

$34.48 \pm 12.8$

$34.74 \pm 7.0$

$34.57 \pm 8.0$

$33.96 \pm 7.5$

Internal/External

Rotation

$6.76 \pm 6.9$

$7.98 \pm 6.7$

$8.03 \pm 8.3$

$2.38 \pm 10.3$

$1.42 \pm 10.9$

$-0.24 \pm 10.9$

107

Table 2. Club Head Kinematics at Impact

\begin{tabular}{cccc}
\hline & \multicolumn{3}{c}{ Grip Type } \\
\cline { 2 - 4 } & Strong & Neutral & Weak \\
\hline Angle $\left(^{\circ}\right)$ & $-1.51 \pm 4.7^{* *}$ & $-2.57 \pm 4.5^{*}$ & $-6.36 \pm 6.9^{*}, * *$ \\
\hline Velocity $(\mathrm{m} / \mathrm{s})$ & $38.2 \pm 3.6$ & $38.6 \pm 4.0$ & $38.0 \pm 3.4$
\end{tabular}

109

$*$, ** indicates significant differences, $P<0.05$, of pairwise comparisons 